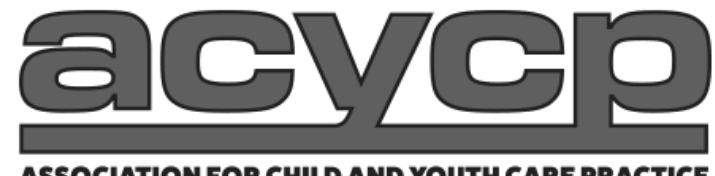

\title{
Staff Experiences of Stress and Coping in a Residential Treatment Facility for Youth
}

\author{
Korri Bickle ${ }^{A}$ \\ AUniversity of Ontario Institute of Technology
}

\begin{abstract}
Working in social services tends to be very stressful for workers. Caregivers are asked to work long hours, complete paperwork and administrative tasks and are responsible for the care and wellbeing of others. Eleven female Childcare Workers were interviewed to determine what causes them stress at work and how they cope. Stress was caused by: long hours, no breaks, low wages, unpaid work, poor communication, consistency and training, high expectations of staff, temper tantrums, abuse, and anxiety about meeting resident's needs. Workers would like more support from their managersand reported a lack of effective teamwork. Many reported not coping well with the stressors of this job and most reported that they take their work stress home with them.
\end{abstract}

\section{Introduction}

Some of the most marginalized and high needs youth live in group care in Canada, the U.S. and the United Kingdom. In Ontario, the Ministry of Children and Youth Services is responsible for organizing residential settings for youth who are not able to reside with their families. Children in Ontario may be placed in a residential setting by the Children's Aid Society, their parents, or through the courts (Ministry of Children and Youth Services, 2016). Residential settings are required to provide accommodation, care and supervision to the residents. In some cases, this care takes place in a foster home, in others it may be in a group home setting operated by staff rather than families. The Ministry of Children and Youth Services notes that services provided to youth in residential settings may include: counselling, behavioural intervention, crisis support, and life skills training, among other things. Group homes in Ontario are operated by service providers who are licenced or funded by the Ministry of Children and Youth Services and these providers are required to meet the regulations of the Child and Family Services Act (Ministry of Children and Youth Services, 2016). Group homes and group home staff play an important role in the welfare of children and youth as they provide care to young people who are often in need of attention and treatment.

As noted previously, many youth are admitted to residential services each year. Jones, Sinha and Trocme (2015) reported that 62,428 of Canada's youth were in out of home care in 2013. In the US, the department of Health and Human Services reports that there were 402,378 children in foster care in 2013. Additionally in 2015, 69,540 children under the age of 18 in England were in care (House of Commons Library, 2015. In January 2016, the Ministry of Children and Youth Services reported that there were 430 children's group homes in operation in Ontario. In order for youth to be receiving the best care, it is important that these group homes provide a safe and effective environment for both children and staff. 
Working in group homes or acting as a foster parent, can be a stressful experience; staff are responsible for the wellbeing of children and youth who often require tremendous amounts of support. It is important that those working in these homes are well trained and committed to doing their best; they are responsible for raising children whose parents are not able to do so. As this paper suggests, high levels of stress can effect staff member's ability to do their best at their job. It is important to recognize where this stress originates from and to develop strategies to reduce it so that workers can focus on giving youth all that they need to grow, and in many instances heal from their experiences.

Those who work in group care facilities are responsible for the wellbeing of others but they often don't feel as though they are in control of their own working lives. This is a job where wages are low and work hours are long, employees are also required to deal with abusive and stressful situations. Staff members in these work places, and others in the human services industry, report that they are stressed at work, but there is little research that examines the sources of this stress. Most research has used quantitative measures that did not directly interview individuals about how they feel, experience or cope with work related stress in group care facilities. Therefore, to extend this important area of research, the current study was conducted via an open-ended qualitative interview process.

\section{Stress in Social Services}

Given the subject matter of this paper, stress that is experienced by people who work in institutional/social service settings is of particular interest. Many of the people who work in settings such as hospitals, residential homes and police and military work have the added stress of working shift work and often receive a lower rate of pay than employees in other fields. Furthermore, these people have more responsibility and less agency or control over their workplace than many other workers.

Stress, has been reported by many employees who work in institutional settings, and who must work with clients/patients who present challenging behaviours. People in this field are often presented with dangerous and anxiety provoking situations. Raczka (2005) conducted a focus group that explored community staff and their experiences of stress while at work. All of the employees in the study noted that they had been involved in at least one situation involving challenging behaviour in the last week. Some experienced feelings of fear and helplessness. Others reported re-experiencing events, and intrusive thoughts following the event. This suggests that these employees are experiencing a level of stress at work that cannot be adequately dealt with using the coping resources that they have available to them.

Hatton et al. (1999) examined factors that are associated with staff stress and satisfaction while working with individuals who have an intellectual disability. They found that general distress and job strain were highly correlated and that these measures were significantly and negatively related to job satisfaction. Results suggested that there are three variables that are associated with general distress in the workplace: wishful thinking; distress associated with work-home conflict; and role ambiguity. Results of this study also indicate that job strain is strongly associated with stress linked to a lack of staff support, alienative commitment, role ambiguity, holding a low status job and longer contracted hours. Lastly, the variables that were strongly associated with job satisfaction were less stress linked to a low status job, support from supervisors, greater influence over work decisions, less alienative commitment, support from colleagues and older staff age. This suggests that when workers have supportive environments around them they function better. It also allows workers to feel they have some agency at work thereby increasing job satisfaction. Further, Jenkins, Rose and Lovell (1997) looked at stress amongst direct-care staff who work with residents with learning disabilities. The authors of this study found that these workers felt as though they were less supported and were significantly more anxious than those staff who worked in residences where there were not the same level of challenging behaviour.

Shinn, Rosario, Morch and Chestnut (1984) were interested in job stress and burnout in human service workers. They found that the areas of the job that were most stressful for this population were excessive workload, role conflict, lack of agency, feelings of inadequacy, interpersonal relations, and emotional demands of both the job and the clients. They also found that job stress was related significantly to three measures of strain; job dissatisfaction, alienation, and psychological symptoms. Huxley et al, (2005) looked at stress experienced by people in mental health social work. They found that feeling under valued at work as well as disagreeing with the way that services are run were reported as stressors for employees. 
People who feel as though they are valued report higher satisfaction with their job. Stress was greatest for those who felt that they did not receive support, and for those who planned to leave their current position. Workers also felt that additional work hours increased stress. Additionally, $47 \%$ of participants had a General Health Questionnaire (GHQ) score that indicated a potential for a psychological disorder. The results of this study suggest that workload and lack of resources can be a major source of stress for workers in this field. Workers who work with individuals that have challenging behaviours or intellectual disabilities often experience great amounts of stress at work. It appears that they have concerns about the amount of work that they have to do and the amount of responsibility placed on them. They do not have control over their environment and they are often involved in dangerous situations. Those who work in the corrections field likely experience similar types of stress.

Many youth who are in the care of the government have also experienced interactions with the youth justice system. Given the overlap that may often exist between youth in custody and youth in care it is appropriate to look at the stress experienced by correctional officers. Lambert, Hogan, Jiang and Jenkins (2009) looked at stress and burnout of correctional staff who worked with youth who had been sentenced as adults. They found that both role ambiguity and role overload were related to staff burnout. The longer that staff worked in a facility the more burnout they experienced. This type of work is very stressful for correctional employees and people in group homes may be experiencing the same amount and type of stressors

\section{Stress Associated with Child and Youth Care Work}

The current study looks at the stress that is experienced by child and youth care workers while working in a specific setting; however, it is important to consider the stress related to this job in any setting. It is imperative to acknowledge that child and youth care workers have struggled with gaining respect from other professionals and from society in general (Barford \& Whelton, 2010). This may lead to additional stress and high turnover rates. In addition, Mattingly (1977) highlighted some of the specific issues leading to burnout and stress for these workers. Workers are responsible for several children at once. They are constantly struggling with managing the children's needs, as well as their own needs, and the reality that they will never be able to give as much as they would like to the children in their care. Further, the rewards of working in this field are inconsistent as workers rarely work with the same youth for long periods. Thus, they may not get to experience or witness the child's success. Workers are dealing with constant verbal, emotional and cognitive stimulation, which can be overwhelming and draining. Mattingly (1977) further recognizes the role conflict that child and youth care workers experience, For example they are responsible for care, custody, and general operations including cooking and cleaning, all the while being intensely aware that they act as an important role model for youth. Finally, child and youth care workers are constantly vulnerable to physical and emotional harm any time during their shift (Mattingly, 1977). Additional stress is related to low wages for highly comprehensive work (Cavaliere 2004).

There have been a number of studies regarding how stressful aspects of the job can lead to burnout. Cordes and Doughery (1993) recognized that age, marital status and the amount of social support the employee believes they have preduct burnout for workers. Further, Barford and Whelton (2010) found that emotional exhaustion in child and youth care workers is related the work environment. This suggests that the work environment can play an important role in the emotional impact of the job and burnout rates. Child and youth care work is mentally, emotionally and physically draining and stressful, it is also a job with few rewards and little recognition. Given what we know regarding the many challenges of front-line staff, it is important to understand what leads to stress and burnout and identify the most effective coping mechanisms for workers.

\section{stress in the Children's Group Home Setting}

The current study aims to understanding the stress that is experienced while working in a group home setting for children. Little effort has been made to understand the sources of stress specifically associated with group home employment. Snow (1994) examined the victimization experienced by the staff members in a group home and the impact of these acts of aggression. Snow's results suggest that following an aggressive incident at work staff experienced increases in nightmares and felt fearful of imminent personal danger. Ninety percent of the sample met the criteria for re-experiencing the trauma and $75 \%$ reported recurrent and intrusive distressing recollections of an incident. The child and youth workers in this study further reported having difficulty falling asleep, increased arousal, increased irritability, outbursts, hyper-vigilance, trouble concentrating, and increased startle response following aggressive incidents at work. Snow (1994) found that meeting these criteria is indicative of psychological distress. Furthermore, the researcher found that $70 \%$ of the sample thought that it was their fault 
that they were injured or assaulted. This suggests that these experiences may cause greater stress group home staff because of their feelings of responsibility and agency.

Robertson et al. (2005) conducted a study that looked at staff satisfaction in community based homes for people with challenging behaviour. Their stress was often related to the lack of resources for dealing with challenging behaviour, the emotional impact of the job, lack of job security, and residents' inappropriate sexual behaviour. When both types of environments were combined (environments with majority of clients having challenging behaviour and minority of clients having challenging behaviour) causes for stress were lack of resources; lack of staff support; work-home conflict; low job status; residents' challenging behaviour; bureaucracy; and poor resident skills. The researchers found that in the combined group the staff members were least satisfied with their rate of pay, followed by opportunities for promotion, management of the organization, recognition for good work, and management-worker relations.

Studies that investigated what causes stress in both child and adult group homes have found many different results but one common area of stress is with co-worker relationships. Rose, Jones and Fletcher (1998) looked at levels of stress in group homes identified by management as either "low" or high "stress" and the apparent differences between the "high" and "low" stress homes. They found that staff in the high stress homes recorded significantly higher levels of general anxiety and higher levels of depression. Significant differences in supports and constraints that were found in the high and low houses were concerned with relationships with others including the relationships with managers, colleagues and visiting professionals. This suggests that staff relationships are quite important and that if staff members perceive that they have more peer supports available to them the amount of stress experienced may be decreased or minimized.

Rose, Jones, and Elliott (2000) conducted a study that looked at different levels of stress between direct care staff and managers in the group home setting; they also looked at perceived levels of pressure and sources of support in these two groups. They found that managers felt significantly more pressure than the direct care staff, however; they also reported greater levels of job satisfaction. Significant differences were reported in the amount of anxiety experienced with managers experiencing more anxiety. The managers in this study reported significantly more sources of work stress than direct care staff and direct care staff reported feeling significantly more support from their line manager than their line managers felt from their superiors. These findings suggest that although direct care workers experience greater client-worker stress there is also a great level of stress experienced at a managerial level that is often related to amount of work and responsibility. Also, managers report a higher level of role ambiguity, this may be a product of managers being promoted from direct care staff; when they get promoted their new role may not be clearly defined, and they may have trouble making the transition due to working closely with others who they now manage.

Johnson (1981) looked at the effect that staff cohesion has on the functioning of a group home. They found that low staff cohesion decreased the amount of staff support, personal problem orientation, practical orientation, autonomy of the clients in the environment and increased the amount of staff control. This suggests that staff cohesion may be important for a well functioning, and effective group home environment. Heron and Chakrabarti (2002) looked into staff's thoughts on provision of care in group homes for children. They found that staff often spoke of being frustrated regarding the amount of time that administrative tasks took away from the children. Staff members also spoke about frustration with knowing what the children needed but not having the resources to meet those needs. Additionally, they reported that frustration at not being involved in decision making, and not having their ideas valued by management and social workers. Workers reported support from other staff members when there was a violent situation; however, with regard to other areas, support was reported to be less common. In terms of control, staff members reported that they felt in control while doing activities with the residents. In other situations, such as when a child becomes violent or a decision is made without their input, the staff members feel as though they are not in control, some even feel disempowerment.

Although there was a high level of stress reported in working in children's homes the majority of the stress came from acts of violence, inadequate debriefing and lack of supervision. Staff reported that stress increased when working a long shift that included staying overnight. During these shifts, the staff reported just "trying to get through" the shift, they reported lack of sleep, trouble unwinding and being expected to start an early shift in the morning. Last, the staff reported that the frustration of not being able to give the children what they needed caused stress. The added stress arose when the children do not get what they needed and reacted with behavioural outbursts 
Staff members in group homes have several sources of stress. They do not have control over their work environment and they are often involved in situations where they feel unsafe or anxious. These workers are asked to do many administrative tasks and this takes away from the residents. Workers in these settings are also asked to work shift work and spend long amounts of time away from their homes. There is a need to further explore stress in the workplace and to identify how people can best deal with work-related stress.

\section{Method}

The study explored stress experienced by staff members working at a residential treatment centre for female victims of physical, sexual, and emotional abuse. Participants in this study were asked about their health, their ideas about control and power at work, the stress that is caused by the shift work and how they felt the organization is operated. Because of the high turnover rate in workers the staff members in this study were looked at in categories organized by the amount of time working within the organization. This categorization is based on the thought that there may be difference in stress and coping for those who stay in the position for longer periods. In addition, staff members who were no longer employed with this facility were considered in order to identify if there were distinct variables that separate the two groups.

\section{Procedure}

Staff members of a residential treatment facility for abused girls were invited to participate in a one-on-one, in depth, semi-structured interview, in accordance with the participants schedule and in a place of their choosing. Consent of the directors of the facility was obtained to conduct this study. The interview protocol consisted of several open-ended questions that probed the workers views on stress and coping both within and outside of the work environment. Staff members were also asked about their views regarding the operation of their workplace, how much agency and solidarity they felt, as well as support they felt they received or did not receive at work.

\section{Interview Participants}

Invitations were extended to all staff members of one residential treatment facility for girls. The sample was comprised of 11, all female, participants. There was a wide range of time spent within the organization and the age of the participants. Participants were recruited by means of verbal invitation and through e-mail. Participation was voluntary and no incentives were offered for participation.

Participants in this study were Childcare Workers at a residential treatment facility for sexually, physically and emotionally abused females. These children had severe psychological consequences because of the trauma that they have experienced and for this reason, the residence is treatment centered. The residents at this facility had lived in several foster homes and group homes before being admitted to the current facility and most had problems with attachment. The staff members supply the residents with constant supervision (unless alone in their bedrooms) and are hyper-vigilante about the interactions between residents to ensure that everyone is safe.

The range of experience of the participants ranged from eight months to twenty-six years.

\section{Analysis}

The researcher used interpretive phenomenology and grounded theory to analyze the data. A phenomenological approach aims to understand the lived experience of several individuals experiencing the same phenomenon at the same time or in the same context. The goal is to note the similarities in experiences, in order to better comprehend the experience at its core (Creswell, 2007). Grounded theory takes this understanding one step further and attempts to generate a theory for understanding the phenomenon. The key is that the theory is not proposed before the research is conducted; the theory is grounded in and emerges from the data (Creswell, 2007). In this study, the transcribed interviews were first coded using open coding in order to discover themes in the data (Van Den Hoonaard, 2012; Creswell, 2007). Next, these themes led to the development of meta-themes (Creswell, 2007). The themes and meta-themes led to the development of the model used to better understand how workers in a residential setting experience stress and coping, as well as why these people experience stress and coping in the ways that they do. 


\section{Results}

The sample was comprised primarily of front line team members, including two team leaders, who work with the residents on a daily basis and are responsible for the day-to-day care of the children. Two individuals who no longer work at the facility, one support worker and two administrative team members were also interviewed. The Team Leaders of the facility are members of the administration. The primary duty of the Team Leaders is the daily care of the residents thus for the purpose of this study they will be referred to as front line staff members unless otherwise specified. At the time of data collection, the facility employed 15 front line workers, 6 administrative team members (including team leaders) and 1 support worker. The entire compliment of staff was approximately 50.

Front line staff members at this facility, who are not team leaders, were expected to work an average of 246.5 hours in a 6 week period or 41.1 hours per week, and 10 overnights in a 6 week period or 1.5 overnights per week. When a front line staff member does an overnight shift they can sleep from 11pm to 7am but these are working hours that are not paid and sleep is often interrupted by residents. Team leaders at this facility were expected to work 230 hours in a 6-week period (38.3 hours per week) and did not have any regularly scheduled overnight shifts. However, one of the responsibilities of the two team leaders is to cover any overnight shifts when a staff member calls in sick or to cover overnight shifts when the facility is short staffed. During the duration of this study, the team leaders were working an average of 6 overnight shifts in a 6 week period or 1 overnight per week.

Most of the members of the administration team were regularly scheduled to work a 9am to 5pm workweek. They had no regularly scheduled overnight shifts and were not obligated to cover for staff members who are ill or otherwise absent. Management expected the administration team to adjust their weekly schedule to cover evenings in the event that there is not enough staff coverage. During the duration of this study each member of the administration team worked an average of 6 overnights, 10 evening shifts, and 5 weekend days in a 6 week period.

The duties of the staff members include completing daily routines with the residents. This may include; supervising morning and evening hygiene, completing routine household maintenance chores, cleaning residents' bedrooms, preparing residents for school, helping them with homework, cleaning the house, making meals on weekends, disciplining the residents, planning and implementing therapeutic activities, delivering therapeutic interventions, doing the residents' laundry (for those who are not able), doing the house laundry (towels, sheets), transporting and accompanying residents on their medical appointments, substitute teaching when teachers are ill, completing night checks at the end of each shift, completing daily notes, completing intervention notes (at least two per day), filling in the daily log, and completing resident notes in order to update staff members coming onto shift.

Front line staff members were also expected to prepare for "plan of care meetings" and clinical case conferences by tracking behaviours and medical concerns, updating the directors on behaviours, improvements in school and daily living, and to present updates to the consulting psychologist at clinical meetings. The Administration team members were the supervisors on site; they were responsible for scheduling, staff discipline and helping out on the floor with the residents when needed.

\section{Workplace Stress is Prevelant in the Workplace}

One of the principle objectives of this research project was to discover the causes of work related stress at this facility. Although there are some forms of stress that are common to many group homes (such as low rates of pay and abuse from residents) there are some sources of stress that may be unique to this facility.

\section{Low rate of pay and no pay for asleep overnight shifts}

Many participants in this study spoke about dissatisfaction with the low rate of pay. In general, participants reported that the amount and level of therapeutic work and care giving work warrants a higher rate of pay. Staff noted that on many of their shifts they were expected to stay late in order to get their paperwork done and they were not able to claim those hours.

Staff members also reported being discouraged because there was little opportunity for promotion within the facility. Participants made it quite clear that they believed they should be paid more money for the work that they do. Staff members thought that they should be paid for the time that they are working at night. They understood that this was supposed to be time for them to be sleeping and preparing for the next day but sleep was often interrupted and they were simply unpaid for working hours. Respondents spoke about the overnight shifts causing 
them anxiety as they were worried about what was going on at home. Often they felt that they had lost two days of their life. This facility had no satellite or cable television, access to the internet was limited and there was no newspaper delivery. This caused staff members to feel disconnected from the outside world.

"I found that working, when you work like a 33 or a 36 hour shift there then it feels like you just kind of missed two days of life, and while your still living but your there so your cut off of everything that is going on in the world and it can be really hard to do that two times a week and then try to still know what's going on in the world and still have a bit of a life and enjoy free time for yourself" (001: 203-207).

Staff members thought that their work environment could be made more ideal if they were either paid for overnight shifts or if new staff were hired specifically for doing the overnights. Participants also suggested that shorter shifts would make their jobs more ideal because they conclude that 33 and 36 hour shifts were too long. This introduces the next theme regarding long shifts and no breaks.

\section{Long shifts and no breaks}

The most common shift in this workplace is "noon overnight to nine", this implies that the staff person comes onto shift at 12 noon one day and stays until 9pm the next night. This shift has the staff member at work for 33 hours. There were also times where this shift was followed by a $12 \mathrm{pm}$ to $9 \mathrm{pm}$ the next day. Staff members discussed this as a major problem, although they appreciated the two days they have off following this shift they spent the majority of their time off on errands and resting for their next shift.

Staff members consistently reported the fact that there hours were too long. They suggested that this was a stressful part of their job because they have to push themselves to make it too the end of their shift. Staff members spoke about not being able to perform at their best level because the shifts are so long that they feel very tired throughout the day (004: 63-64). Participants also spoke about not being able to leave when their shift came to an end. This was a stressor for many staff members as it created problems with their families when they stayed late at work. This conflict with staying late at work caused staff members to be unsatisfied with their jobs. Childcare Workers discussed the fact that they should be able to have scheduled breaks. They believed that with the long hours they worked it would be beneficial for them to have some time to spend on their own. Many people talked about working hard from the minute they walked in the door and they did not get enough time to regroup. Although there were no specific rules stating that staff members were not able to have breaks the general idea that the staff members got is that if you are not a smoker you don't get a break. There was also a sense that if you are "caught" taking a break others were going to think that you were slacking. Many front line workers who do not smoke thought that the smokers were taking too many breaks and noted that they thought this was not fair. This is connected to the next theme of high expectations and a heavy workload for staff members.

\section{Heavy workload and high expectations of staff}

Staff members within this facility concluded that they were expected to do too much in the amount of time that they were given. Many staff members noted that there was not enough time to do these tasks on top of the high level of supervision that they need to provide for the residents.

"I think that too much, the workload on each individual person is too much that, um, everybody gets so burnt out that you, you can't do it anymore" (005: 104-105).

The team leaders and the members of the administration team believed that extra work was put on them because they are often expected to be on the floor when they should be doing other tasks. They also seemed to feel that they had too many roles. One of the team leaders talked about feeling stressed because many clinical pieces are given to her to do and she didn't have enough time to get them all done (004: 113-116). Also, the members of the administrative team reported that there is not enough time to focus on their job because they were spending a lot of time working on the floor with the residents. They also felt stressed because usually when their shift was over they couldn't go home (008: 290-292). 
Front line staff spoke about the issue that they were supposed to do many jobs at once and that their role at work was not clearly defined.

"there's really no definition of what your job is, your just kind of expected to understand and do it all, and there's positions where your supposed to do one thing but your expected to do another job at the same time" (005:106108).

Staff members thought that they were expected to do more than they can reasonably complete in the time that was provided for them at work. They reported that they were expected to know what to say and how to act according to each child's history and trauma triggers. They also discussed several issues that made this goal hard to achieve. Staff members did not have adequate training to be able to meet expectations. Front line workers also talked about the problem that there was not enough communication between staff members and that consistency with the resident's programs was also a problem.

\section{Staff training, communication and consistency}

Respondents revealed that there were a number of issues with staff communication at this facility. Although the Clinical Director was available by phone this didn't seem be the type of training the staff needed. This method of communication tended to be used in order to get questions answered but was not adequate as an ongoing training tool. The Clinical Director was willing to provide supervision time to staff members however it needed to be on a staff member's days off and it was unpaid. Several staff members suggested that if the Clinical Director was at the group home more often it would have allowed for more training, better consistency and a greater level of communication. More experienced staff members felt stressed because new staff members were required more training. They worried that the residents were not getting what they needed from new staff and felt pressured to do more of the emotionally and physically draining work.

Participants suggested that one of the large problems within this facility was a lack of communication between staff members. They talked about not being able to do their job properly because they were not provided with all of the information needed at the beginning of their shift. Participants talked about not being able to do their best for the residents because of staff communication problems. Staff members spoke a fair amount about how important it was for them to be consistent in their job because this was something that was very important for the daily functioning of the residence. A high level of communication was the only way that staff members could make sure they were being consistent.

\section{Restraints, temper tantrums, and abuse}

Staff members at this facility reported that the major job stressors were the temper tantrums that they dealt with as well as verbal and physical abuse from the residents. Although many of the staff members understood that these children were in crisis during their temper tantrums they also noted that it was a part of their job that made them feel anxious. Staff members spoke about restraints being hard for them because they often felt upset afterwards. Some staff worried that they had done something incorrectly or that they could have done something differently in order to avoid the need for physical restraint. Other staff members felt sympathy for the residents or had a hard time debriefing.

"more the stress of afterwards, of after restraining a kid, going oh my goodness I just had to restrain this kid, and its not so much feeling stress as on the kid, its on yourself because really you know, um, its one of those, did I do something wrong, could I have like, not really wrong, could I have changed it so that I didn't have to restrain the kid and then you like, stress about did I do it right, that kind of thing" (007: 216-220).

Respondents struggled with the verbal abuse that was often involved in a temper tantrum. Workers wanted to react to the situation in the appropriate manner but they weren't always certain that their response was correct and this in itself was a source of stress.

Overall, the staff members believed that their job was very emotionally and physically draining and that temper tantrums and abuse only added more stress. Further, the staff members at this facility believed that it was very 
important to do the best that they could for the residents in terms of teaching them life skills and preparing them for their future. Though there was no expectation from management to do this, staff felt stress about how prepared the residents were. They did not want them to go out into the "real world" without being prepared.

\section{Meeting the needs of residents}

A number of the staff members worried that the residents left the facility without a true concept of what the "real world" was like. Staff members said that they thought residents were not being adequately prepared for what their lives were going to be like outside of the group home. Although workers realized that the residents needed to feel safe they worried about shielding the residents too much from the outside world. Staff members worried that the residents would struggle once they were discharged from the facility.

Staff members also worried about meeting all of the needs of the residents. They talked about feeling stressed or anxious when they didn't know what to do to help the residents. Some participants worried about helping the residents deal with their historic abuse before they left the residence. Staff members said that this insecurity about not preparing the residents and providing them everything they needed to succeed was a major source of stress.

"I would say the main thing you stress about is, um, just when a kids going through that stage where we're just standing still with them, when their out of control and we don't really know what to do to help them, I think (a resident is) there, that's stressful I don't like that step" (010: 150-152).

The staff members at this facility worked hard to do their best for the children but they were still left wondering if they were doing the best they could. As discussed, there are many causes of stress in this field. This paper will now turn to how these workers were dealing with there work related stress.

\section{Teamwork and Support}

Staff members at this facility needed to be supported on a regular basis due to the amount of stress that they experience. When staff members were asked about support and teamwork at the facility it seemed to depend on how long the individual had worked at the facility. Most people felt that there was support and teamwork to some extent; however, the degree of each varied based on experience. Those who had worked at the facility for a relatively short time, and those who had worked at the facility for a long time appeared to hold similar views. Staff members who had worked at the facility for less than two years generally reported that they received a lot of support from their co-workers. Similarly, the staff members who had worked at this facility for a long time believed they were supported in their workplace. These staff members tended to believe there was a strong team at the time of the study.

The staff members who had worked at this facility for an intermediate period however, namely between two and 6 years seemed to believe that support in the workplace was limited and teamwork could've been better. Some of the staff members reported that people who work in this facility created little cliques, rather than working all together and they spend time talking about others behind their backs. In terms of support, this group tended to think that there was support from team members; however, they did not receive as much support from the Directors.

Although there were some staff members in this category who felt quite supported, they seemed to be in the minority. Staff members in this group talked about how the support was there some of the time, however it was not in all areas, and a lot of the time they needed to seek out support. There was some controversy in this area, however the majority of the group tended to believe that there was not very much support and that the teamwork that existed needed to be improved.

\section{Discussion}

\section{Stress}

The results of this study suggest that stress plays an important role in this workplace. Staff members at this facility felt that there were a number of factors that contributed to their levels of stress at work. As expected, 
they believed that they were paid too little for the work that they do and that the long hours and expected overtime without remuneration were causes of stress both at work and at home. They felt that their stress often came home with them and interfered with their days off. Similar to Robertson et al. (2005), results suggest that low rate of pay was a contributor to job stress. Staff felt low pay was also related to high staff turn-over, staff believed that it would be simpler to go find another job that paid more and was less stressful so incentive to stay was low. Participants also suggested that overnights were a difficult aspect of the job. The fact that they were not paid for overnights made it even more stressful. In this particular setting the staff felt that the shifts were too long and it took away from their ability to work effectively with the residents. Not only did staff report feeling as though they missed two days of their lives, but the shifts seemed to interfere with their ability to enjoy their time off, as if they were always playing "catch up".

Staff members felt that there was little support in their positions, though there was variation in this belief. Workers who were recently employed or who had been long term employees reported feeling more support. Those who had been employed for more than 5 years tended to feel that there was personal, as well as professional support suggesting that the relationship that they had built with the directors may have played an important role in their experience of support. The majority of front-line workers felt that there was a lack of support from their teammates as well as from the directors, similar to the findings of Jenkins, Rose and Lovell (1997) who reported that individuals who worked in homes with challenging behaviour felt that they were less supported than workers in other homes. This lack of support is a concern as the literature suggests that when individuals feel that they have more peer support their level of stress is decreased or minimized (Rose, Jones \& Fletcher, 1998). This is an important finding as increasing peer support can act as a protective factor for the level of stress experienced by staff in this setting.

In addition to the perceived lack of support and teamwork, there was concern about the lack of communication and consistency among staff members. With the high needs of many residents there were individual programs created for each youth that changed regularly. When the program was not executed properly, the residents became frustrated and the staff often felt disempowered. The residents seemed to know more about the program than staff did. Staff discussed the stress that was associated with others not communicating program changes and staff members who knowingly did not follow a resident's program.

Staff members at this facility also mentioned that they worried about meeting the needs of the residents in terms of preparing them for life on the outside. They appeared to have a real interest in helping these youth; however, they were struggling with whether or not they were able to achieve this goal. It might be useful for staff members to have these discussions in an open and honest way during team meetings. It is possible that their expectations for some of these residents, and their ability to prepare them, are beyond what is possible within the limits of facility resources. However, it is also possible that there are ways that they could improve their strategies for preparation. For example by including additional programming and daily living skills. It would be useful for these staff members to feel that they have a forum for discussing their fears and input regarding ways to improve their work.

Further, in keeping with studies in similar settings, these staff members reported physical restraints and temper tantrums as being a very stressful aspect of their job. These reports of stress were comparable to the intrusive thoughts that staff reported experiencing after a behavioural event in a study by Raczka (2005) and the recurrent and distressing recollections of these types of incidences found by Snow (1994). Staff in this study worried about saying the right things and second-guessing their decisions hoping that they didn't do anything that could have escalated the situation. They felt an extreme sense of responsibility in terms of trying to provide a safe and trauma aware environment while still providing residents with the opportunity to grow and develop in a way that would prepare them for "the real world". Concern for safety, general wellbeing and adequate preparation is likely an important characteristic of staff members in these facilities; however, it is important that there is support and training in place for staff to ensure that these worries do not interfere with their ability to do their job.

Finally, consistent with research in this area, it appears that the staff members in this facility, (Rose, Jones \& Elliot, 2000), experienced role ambiguity. They mentioned that they were expected to maintain a high level of supervision, captain other activities, clean the house, cover for school staff, plan activities etc. and that there was no definition for their role. Research suggests that this can be a cause of stress in similar work environments and it appears that it plays a role in this residence as well. Administrative team members also struggled as they were expected to work front line with residents as well as completing various other tasks and there was not enough time for them to do their proscribed job. 


\section{Coping}

Coping strategies were an important focus of the study interviews. There did seem to be some differences in the ability to cope within the staff group. Staff reported coping with work stress in a number of ways, and recognized that many of the strategies used were more effective and healthier than others. They reported taking work stress home with them by thinking and talking about work when not on the job. Staff noted that their family members didn't want to talk about work but staff still felt as though they needed to, causing issues at home. Other staff members felt that keeping their stress bottled up inside was the only way to deal with it, they didn't want to discuss it with others or felt that there was nothing that could be done about it.

There were some individuals who had more effective coping strategies, these included talking with team members at the end of their shift, working together to problem solve and trying to look at issues from another perspective. Another strategy was trying to get a moment for themselves during the day. Although some people found that the only way that they could do this was going to for a cigarette break, they did feel that getting these few minutes helped them to regroup and feel refreshed. Those who did not smoke mentioned trying to get a few minutes in the staff room or going for a walk. For those people who are not able to leave their stress behind, some had some good strategies for reducing their stress on their way home (talking it out with themselves or listening to music) or taking some time when they got home to have a bath or a cup of tea to relax and de-stress.

There did seem to be some differences between those who were able to leave their stress at work and those who struggled with it. Staff who were able to leave stress at work were those who did not have to do overnight shifts and in general had shorter shifts (8-10 hours). In addition these tended to be the people who used problem-solving strategies to deal with stress rather than just venting or keeping it in. Those who took work home with them worked overnights and longer shifts (24-36 hours). These staff also tended to have family members that were not supportive of the type of work that they do and the length of their shifts. These findings suggest that it is possible for staff members in this facility to leave their work stress at work but there are a number of factors that contribute to the success of this. Though it should be noted that the length of shifts and inclusion of overnights was not within the staff's control. If it were, perhaps staff may have been able to develop more effective problem solving strategies that would help them to feel less stressed when they left work.

\section{Limitations and Conclusions}

There may be some concern with the fact that the principle investigator also worked at the facility where this research was conducted. It may have limited participant's involvement in the study and the depth of participant disclosure. However, the researcher's experience in the facility contributed to her ability to understand the dynamics of the organization and develop a more informed research design. Further, participants seemed to feel more comfortable with sharing information as the researcher already had a rapport with these individuals. Although there may have been some concerns about the principle investigator working at the facility it could be argued that the limitations of this were outweighed by the benefits. A further limitation of this study is that all participants worked at the same residence. Although this provides rich knowledge regarding their experience, it may limit the extent to which the results are generalizable to other organizations. The findings from this can be generalized to similar organizations; however, they cannot be applied to all child and youth workers.

The current research has provided important information about staff members working in a group home setting and the stress that they experience. Some of the results of this study are similar to other studies such as workers being stressed about the amount of paperwork they have to do and the worry about meeting the needs of residents. There were also some results that are new to this area. For example, the study found that smokers taking breaks, overnight shifts and lack of communication are important sources of stress for these individuals. Of concern is the fact that not all staff members feel supported in these high stress environments and that teamwork may be a problem.

Given the results of this study, it is important to think about what can be done to reduce staff sources of stress. Overnight shifts cause a great amount of stress to these individuals and operators need to think about other ways to manage their schedules so that staff members have shorter shifts and more breaks. It may also be beneficial for staff members to be involved in team building exercises so that people can learn to work together and support each other when needed. Lastly, lack of communication between workers in all areas of an organization such as 
this is an enormous cause of stress. Operators of these facilities need to develop some way for workers to have better ways to communicate.

In order for workers to feel supported and safe at work there needs to be an environment where people feel comfortable voicing their concerns. Staff members need to feel supported in their work environment and they have to develop strategies to cope with stressors throughout the day in order for them to do their jobs properly. If operators can work with their employees to discover what their sources of stress are and help diminish even a small part of it, better care would be provided to residents and burnout of workers might be decreased.

\section{References}

Barford, S.W. and Whelton, W.J. (2010) Understanding burnout in child and youth care workers.

Child \& Youth Care Forum, 39, 271-287.

Cavaliere, G. (2004). Cream city dreams: Reflections on professionalization of child and youth care workers in the US. Child and Youth Care Forum 33(6), 375-378.

Cordes, C. L., \& Dougherty, T. W. (1993). A review and an integration of research on job burnout. Academy of Management Review, 18(4), 621-656.

Creswell, J. W. (2007). Qualitative inquiry \& research design: Choosing among five approaches. United Kingdom: SAGE Publications.

Hatton, C., Emerson, E., Rivers, M., Mason, H., Mason, L., Swarbrick, R., et al. (1999). Factors associated with staff stress and work satisfaction in services for people with intellectual disability. Journal of Intellectual Disability Research, 43(4), 253-267.

Heron, G., \& Chakrabarti, M. (2002). Examining the perceptions and attitudes of staff working in community based children's homes: Are their needs being met? Qualitative Social Work: Research and Practice, 1(3), $341-358$.

Van Den Hoonaard, D. (2012). Qualitative research in action: A Canadian primer. Don Mills,

Ont: OUP Canada.

Huxley, P., Evans, S., Gately, C., Webber, M., Mears, A., Pajak, S., et al. (2005). Stress and pressures in mental health social work: The worker speaks. British Journal of Social Work, 35(7), 1063-1079.

Jenkins, R., Rose, J., Lovell, C. (1997). Psychological well-being of staff working with people who have challenging behaviour. Joumai of Intellectual Disability Research, 41(6), 502 -

511.

Johnson, S. M. (1981). Staff cohesion in residential treatment. Journal of Youth and Adolescence, 10(3), $221-232$.

Jönsson, D. S., Johansson, S., Rosengren, A., Lappas, G., \& Wilhelmsen. (2003). Self-perceived psychological stress in relation to psychosocial factors and work in a random population sample of women. Stress and Health, 19(3), 149.

Lambert, E. G., Hogan, N. L., Jiang, S., \& Jenkins, M. (2009). I am fried: Stressors and burnout among correctional staff. Corrections Compendum, 34(2), 16-23.

Mattingly, M. A. (1977). Sources of stress and burn-out in professional child care work. Child and Youth Care Forum 6(2), 127-137.

Ministry if Children and Youth Services. (2016). Because Young People Matter: Report of the Residential Services Review Panel.

Ministry if Children and Youth Services. (2016). Residential Service. Retrieved November 1, 2016 from http://www.children.gov.on.ca/htdocs/English/professionals/childwelfare/ residential/index.aspx

Poole, G., Hunt-Matheson, D., \& Cox, D. N. (2005). The psychology of health and health care (2nd ed.). Toronto: Pearson Education Canada Inc.

Raczka, R. (2005). A focus group enquiry into stress experienced by staff working with people 
with challenging behaviours. Journal of Intellectual Disabilities, 9(2) 167-177.

Reichenberg, A., \& MacCabe, J. H. (2007). Feeling the pressure: Work stress and mental health. Psychological Medicine, 37(8), 1073-1074.

Robertson, J., Hatton, C., Felce, D., Meek, A., Carr, D., Knapp, M., et al. (2005). Staff stress and morale in community-based settings for people with intellectual disabilities and challenging behaviour: A brief report. Journal of Applied Research in Intellectual Disabilities, 18(3), 271-277.

Rose, J., Jones, C., \& Elliot, J. L. (2001). Differences in Stress Levels between Managers and

Direct Care Staff in Group Homes. Journal of Applied Research in Intellectual Disabilities, 13(4), $276-282$.

Rose, J., Jones, F., \& Fletcher, B. C. (1998). The impact of a stress management programme on staff well-being and performance at work. Work and Stress, 12(2), 112-124.

Snow, K. (1994). "Aggression: Just part of the job?": The psychological impact of aggression on child and youth workers. Journal of Child \& Youth Care.

Shinn, M., Rosario, M., Mørch, H., \& Chestnut, D. E. (1984). Coping with job stress and burnout in the human services. Journal of Personality and Social Psychology, 46(4), 864-876.

fermentum posuere finibus. Ut cursus lorem nibh, id bibendum tortor elementum et. Proin eget lacus mi. Mauris scelerisque sed ipsum eu vulputate.

Quisque porta sollicitudin aliquet. Praesent sit amet feugiat eros. Nulla vulputate, mauris scelerisque ornare porta, odio nibh luctus nunc, et tempus ligula ante eu felis. In auctor purus in dignissim dapibus. Proin at eros enim.

Phasellus id rhoncus nibh. Morbi dapibus venenatis interdum. Cras elementum orci magna, sed placerat magna interdum eu. Phasellus dictum facilisis ante.

Duis auctor, neque ut ultrices suscipit, orci ex mattis odio, at eleifend sem eros a mauris. Nulla tellus massa, tincidunt ut dictum varius, auctor sed quam. Sed tempor tempus justo, semper ullamcorper urna aliquam eu. Aenean imperdiet vehicula molestie.

Cras et ligula lacinia, lobortis quam eu, maximus nulla. Nam at ex a ex rhoncus dignissim. Suspendisse non quam dignissim purus egestas bibendum. Sed sed convallis nulla, nec auctor mi. Cras ligula velit, iaculis nec nibh sit amet, ullamcorper elementum felis.

Duis suscipit, magna sit amet bibendum scelerisque, augue tortor venenatis mauris, nec aliquam magna tortor ac quam. 